\title{
Fuzzy Predictive Control of Wind Turbine Systems Via Singular Perturbation Theory
}

\author{
Yan Zhang, Zhong Yang, Haifei Si \\ Jinling Institute of Technology, 211169 Nanjing, China
}

\begin{abstract}
In this paper, the maximum power point tracking problem of variable-speed wind turbine systems is studied. The mechanical and electromagnetic dynamics of the wind turbine systems are both taken into account. Since the electromagnetic parts change much faster than the turbine part, the whole system is modeled in two time scales base on singular perturbation theory. And a T-S fuzzy model predictive control strategy is then developed. The controller is validated with a wind turbine simulator. The results have shown better performance in comparison with existing controllers.
\end{abstract}

\section{Introduction}

Wind energy is a clean and inexhaustible resource. Nowadays wind conversion control systems have attracted lots of researchers' attention, especially about variable-speed turbines.

Variable-speed wind turbines operate in two regimes, below-rated power and above rated power. Control objectives of wind conversion are different depending on the operating area. For low wind speed, the primary aim is to maximize the wind energy capture [1], [2]. Many research work are in the aim to optimize wind power capture [2]-[5]. This paper focuses on rotating the rotor speed at a reference proportional to the effective wind speed in order to reach the maximum wind energy capture. In other words, the maximum power point tracking problem is considered in this paper.

Many control strategies, including linear and nonlinear controllers, were proposed in the existing literature. PID pitch controller is a classical approach for the rotor speed, which can be found in [6]. Another known method is an LQG controller [7], [8] These controllers resulted in acceptable results, but still insufficient. Then, intelligent control ideas are combined with the classical approaches. References [9], [10] present fuzzy PID controller to capture the maximum wind energy. And references [11] and [12] employ neural network and genetic algorithm respectively for maximum power extraction.

As for the nonlinear control methods, Boukhezzar ${ }^{[13]}$ dissociates the generator and aeroturbine control, designs a nonlinear cascaded control structure containing two control loops: the inner loop deals with the electric generator and the outer loop concerns the aeroturbine. In [14], a nonlinear model predictive controller is developed. The predictive calculation is based on the input-output feedback linearization scheme, and the control law is derived from optimization of an objective function.

Although a considerable bibliography is devoted to the maximum power point tracking problem, most of them do not consider that the generator part varies much faster than the aeroturbine part. However, as a matter of fact, mechanical components are slower compared with electrical components. The whole wind turbine system is in two-time-scale. It is known that two time scale system usually are difficult to control. A slow controller is impossible to respond the fast states timely and appropriately. As for a fast controller, the variation of the slow states is nearly zero in a short time, and this will lead to data loss.

Singular Perturbation (SP) theory is a "gift" to control these multiple scale systems [15], [16] with the remedial features of both dimensional reduction and stiffness relief. In this paper, the wind turbines' mechanical and electrical components are both exploited, in a nonlinear singularly perturbed model. Then, the mathematical model is reformed into T-S fuzzy model. A T-S fuzzy model based predictive controller is proposed.

The contribution of this paper is, on the one hand, the consideration of both mechanical and electrical components. Taken the wind rotor speed $\omega_{r}$, generator speed $\omega_{g}$ and internal torque $T_{H}$ as the slow states, and $q, d$ components of the stator currents, $i_{d}, i_{q}$ as the fast states, a singularly perturbed T-S fuzzy model is presented. With regards to the literature, this model takes advantage of the two-time-scale property of the wind turbine systems.

On the other hand, the contribution consists of proposing a nonlinear Model Predictive Controller (MPC) composed of slow and fast MPC sub-controllers based on the T-S fuzzy model. The cost function is defined of tracking error $e(t)$ (between the rotor speed $\omega_{r}$ and the 
optimal rotor speed $\omega_{r}^{r e f}$ ) and the variation of the input, $\dot{u}(t)$. By the optimization of the cost function, a subcontroller is derived to maintain the rotor speed $\omega_{r}$ at the optimal rotor speed $\omega_{r}^{\text {ref }}$. In doing so, the wind energy capture is minimized. Similarly, the fast MPC subcontroller could be designed. And the whole controller is composed of slow sub-controller and fast sub-controller.

The remainder of this paper is organized as follows. The singularly perturbed T-S fuzzy model of the wind turbine systems is described in Section 2. Section 3 designs a composite model predictive controller. In Section 4, the control strategy is validated upon the mathematical model using MATLAB. In the end, Section 5 discusses the conclusion of this work.

For the reader convenience, the list of symbols is given in Table 1.

Table 1. List of symbols.

\begin{tabular}{|c|c|}
\hline Symbols & Meaning \\
\hline$V(t)$ & Wind speed, $m s^{-1}$ \\
\hline$\omega_{r}(t)$ & Rotor speed, $\mathrm{rads}^{-1}$ \\
\hline$\omega_{\text {ref }}(t)$ & Optimal rotor speed, $\mathrm{rads}^{-1}$ \\
\hline$\omega_{g}(t)$ & Generator speed, $\mathrm{rads}^{-1}$ \\
\hline$T_{H}$ & Internal torque, $\mathrm{Nm}$ \\
\hline$i$ & Gearbox ratio \\
\hline$\eta$ & Gearbox efficiency \\
\hline$J_{r}$ & Wind rotor inertia, $\mathrm{Kg} \mathrm{m}^{2}$ \\
\hline$J_{g}$ & Generator inertia, $\mathrm{Kg} \mathrm{m}^{2}$ \\
\hline$K_{g}$ & $\begin{array}{l}\text { High-speed shaft stiffness } \\
\text { coefficient, } \mathrm{Nmrad}^{-1}\end{array}$ \\
\hline$B_{g}$ & $\begin{array}{l}\text { High-speed shaft damping } \\
\text { coefficient, } \mathrm{Kg} \mathrm{m}^{2} \mathrm{~s}^{-1}\end{array}$ \\
\hline$P$ & Number of pole pairs \\
\hline$\Phi_{m}$ & Flux linkage, $W b$ \\
\hline$i_{d}$ & $\begin{array}{c}d \text { components of the stator } \\
\text { currents, } A\end{array}$ \\
\hline$i_{q}$ & $\begin{array}{c}q \text { components of the stator } \\
\text { currents, } A\end{array}$ \\
\hline$T_{r}$ & Aerodynamic torque, $\mathrm{Nm}$ \\
\hline$R_{s}$ & Stator resistance, $\Omega$ \\
\hline$L_{d}$ & $\begin{array}{c}\text { Stator } d-\text { axis inductance, } \\
m H\end{array}$ \\
\hline$L_{q}$ & $\begin{array}{c}\text { Stator } q-\text { axis inductance, } \\
m H\end{array}$ \\
\hline
\end{tabular}

\begin{tabular}{|c|c|}
\hline$u_{d}$ & Stator $d-$ axis voltage, $V$ \\
\hline$u_{q}$ & Stator $q-$ axis voltage, $V$ \\
\hline
\end{tabular}

\section{System Description Decomposition \\ 2.1 Problem Description}

Consider a class of wind turbine systems developed as a nonlinear singularly perturbed model as below:

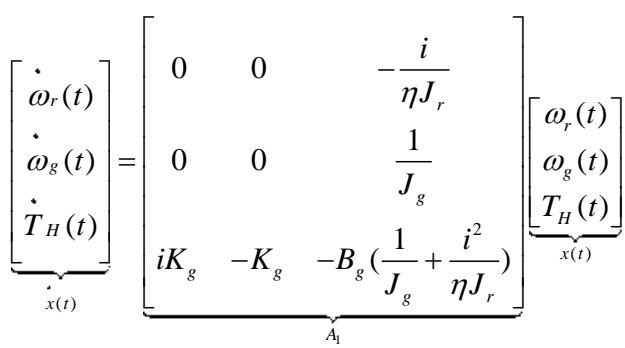

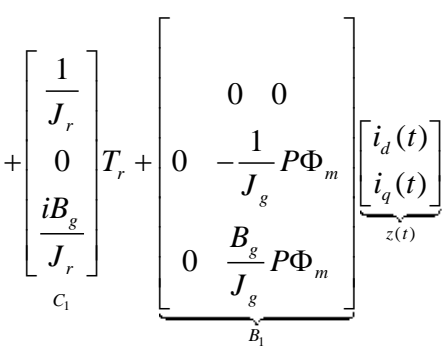

$$
\triangleq A_{1} x(t)+B_{1} z(t)+C_{1} T_{r}
$$

$$
\begin{aligned}
\dot{\varepsilon} \dot{i}_{d}(t) & =-\frac{R_{s}}{L_{d} \times 10^{2}} i_{d}(t)+\frac{P L_{q}}{L_{d} \times 10^{2}} i_{q}(t) \omega_{g}(t) \\
& -\frac{1}{L_{d} \times 10^{2}} u_{d}(t)
\end{aligned}
$$

and

$$
\begin{aligned}
\varepsilon \dot{i}_{q}(t)= & -\frac{R_{s}}{L_{q} \times 10^{2}} i_{q}(t)-\frac{P}{L_{q} \times 10^{2}}\left(L_{d} i_{d}(t)-\Phi_{m}\right) \\
& \times \omega_{g}(t)-\frac{1}{L_{q} \times 10^{2}} u_{q}(t)
\end{aligned}
$$

where $\varepsilon=0.01$. Define $z=\left[\begin{array}{ll}i_{d} & i_{q}\end{array}\right]^{T}$, and rearrange equations (2) into the matrix form as below

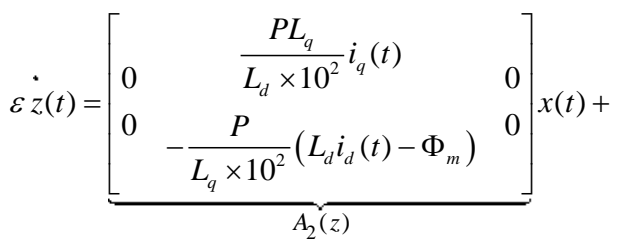

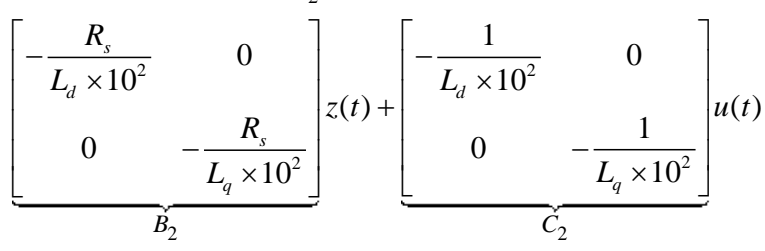

$\triangleq A_{2}(z) x(t)+B_{2} z(t)+C_{2} u(t)$ 
So we get the system expressed as the singularly perturbed model:

$$
\begin{aligned}
& \dot{x}(t)=A_{1} x(t)+B_{1} z(t)+C_{1} T_{r} \\
& \dot{\varepsilon} \dot{z}(t)=A_{2}(z) x(t)+B_{2} z(t)+C_{2} u(t)
\end{aligned}
$$

Our objective is to rotate the wind rotor speed $\omega_{r}$ to track the optimal value $\omega_{r}^{\text {opt }}=\lambda_{\text {opt }} V(t)$. Further more, the system will be presented in a fuzzy linear dynamic model called Takagi-Sugeno model. This fuzzy model is described by the IF-THEN rules, and the $j$ th rule is of the following form:

Plant Rule $j$ : IF $i_{d}(t)$ is of $M_{j 1}$ and $i_{q}(t)$ is of $M_{j 2}$

$$
\text { Then }\left\{\begin{array}{c}
\dot{x}(t)=A_{1} x(t)+B_{1} z(t)+C_{1} T_{r} \\
\dot{\varepsilon} \dot{z}(t)=A_{2 j} x(t)+B_{2} z(t)+C_{2} u(t)
\end{array}, \quad j=1,2, \cdots r .(5)\right.
$$

where $M_{j 1}$ and $M_{j 2}$ are the fuzzy term-set, $r$ is the number of IF-THEN rules, and $i_{d}(t)$ and $i_{q}(t)$ are the premise variables.

Then, the overall singularly perturbed model is inferred as follows:

$$
\begin{aligned}
& \dot{x}(t)=\sum_{j=1}^{r} h_{j}\left\{A_{1} x(t)+B_{1} z(t)+C_{1} T_{r}\right\} \\
& \dot{\varepsilon} \dot{z}(t)=\sum_{j=1}^{r} h_{j}\left\{A_{2 j} x(t)+B_{2} z(t)+C_{2} u\right\}
\end{aligned}
$$

where

$$
\begin{aligned}
& h_{j}=\frac{\mu_{j}(z(t))}{\sum_{j=1}^{r} \mu_{j}(z(t))}, \quad \mu_{j}(z(t))=M_{j 1} M_{j 2}, \\
& \left\{\begin{array}{l}
\sum_{j=1}^{r} \mu_{j}(z(t))>0, \quad j=1,2, \cdots r \\
\mu_{j}(z(t)) \geq 0
\end{array}\right.
\end{aligned}
$$

For all $t, h_{j}$ is the grade of membership of $z(t)$ in $M_{j l}$. From the definition of $h_{j}(z(t))$, it is easy to be seen that

$$
\left\{\begin{array}{l}
\sum_{j=1}^{r} h_{j}(z(t))=1 \\
h_{j}(z(t)) \geq 0
\end{array}, \quad j=1,2, \cdots r\right.
$$

Remark 1: Since the $A_{2}(z)$ varies with time $t$ and the state $z$, it is difficult to get the unique solution of $z$ as $z_{s}=h\left(t, x, u_{f}\right)$ for order reduction from equation (4). To make up for this drawback, we linearized the nonlinear model based on the different values of $A_{2}(z)$ while $z(t)$ changes. Namely, the fast state $z(t)=\left[\begin{array}{ll}i_{d}(t) & i_{q}(t)\end{array}\right]$ is the premise variable.

Remark 2: By representation of the singularly perturbed nonlinear dynamic model of the wind turbine system, we have obtained a linear dynamic fuzzy model. For a linear model, much more practical linear control theories can be applied. In this paper, we adopt the Model Predictive Control (MPC) method.
Remark 3: It can be noticed that in the equation (6), the coefficient matrices do not vary with the subscript $j$. That is, $\left\{A_{1} x(t)+B_{1} z(t)+C_{1} T_{r}\right\}$ part is independent of $h_{j}$. Therefore, the equation (6) can be simplified into $\dot{x}(t)=A_{1} x(t)+B_{1} z(t)+C_{1} T_{r}$. However, to keep the uniform formats of the slow and fast subsystems, we prefer the equation (6) to be expressed of the fuzzy model.

As we can see that the singular perturbation parameter $\varepsilon$ is caused because of the different time scales between the mechanical dynamic, namely the speed and torque, and the electric dynamic which are the varying electric current. With the small parameter $\varepsilon$ multiplying the state $z$, it is difficult to apply common analysis and control theories to the SPS.

In order to analyze and control the singular perturbed T-S model (6), the corresponding slow and fast subsystems are derived.

\subsection{Two-Time-Scale System Decoupling}

In this section, the system decoupling procedure is briefly described of equations $(5)^{[12,17]}$.

Define new variables as $\eta=z+L_{j} x, \xi=x-\varepsilon N_{j} \eta$, then we can get

$$
\left[\begin{array}{c}
\dot{\xi} \\
\dot{\varepsilon}
\end{array}\right]=\left[\begin{array}{cc}
F_{1 j} & 0 \\
0 & F_{2 j}
\end{array}\right]\left[\begin{array}{l}
\xi \\
\eta
\end{array}\right]+\left[\begin{array}{l}
G_{1 j} \\
G_{2 j}
\end{array}\right] T+\left[\begin{array}{c}
H_{1 j} \\
H_{2 j}
\end{array}\right] u
$$

where $F_{1 j}=A_{1}-B_{1} L_{j}, F_{2 j}=B_{2}+\varepsilon L_{j} B_{1}$, $G_{1 j}=C_{1}-\varepsilon N_{j} L_{j} C_{1}, G_{2 j}=\varepsilon L_{j} C_{1}, H_{1 j}=-N_{j} C_{2}, H_{2 j}=C_{2}$. $L_{j}$ and $N_{j}$ satisfy

$$
\begin{array}{r}
A_{2 j}-B_{2} L_{j}+\varepsilon L\left(A_{1}-B_{1} L_{j}\right)=0 \\
N_{j} B_{2}-B_{1}+\varepsilon N_{j} L_{j} B_{1}-\varepsilon A_{1}+\varepsilon B_{1} L_{j} N_{j}=0
\end{array}
$$

First, the jth plant rule of fuzzy slow subsystem is expressed as below:

Plant Rule $j$ :

$$
\text { IF } i_{d}(t) \text { is of } M_{j 1} \text { and } i_{q}(t) \text { is of } M_{j 2}
$$

THEN

$$
\dot{\xi}(t)=F_{1 j} \xi(t)+H_{1 j} u_{s}(t)+G_{1 j} T_{r}
$$

where $A_{01 j}=A_{1}-B_{1} B_{2}^{-1} A_{2 j}$, and $B_{01}=B_{1} B_{2}^{-1} C_{2}$.

Second, the $j$ th plant rule of fuzzy fast subsystem is expressed as below

Plant Rule $j$ :

IF $i_{d}(t)$ is of $M_{j 1}$ and $i_{q}(t)$ is of $M_{j 2}$

THEN

$$
\dot{\varepsilon} z_{f}(t)=B_{20} z_{f}(t)+C_{20} u_{f}
$$

where $B_{20}=B_{1} B_{2}^{-1}$. 
Define $\tau=\frac{t}{\varepsilon}$, and substitute it into the fast subsystem, then we can obtain

$$
\frac{d z_{f}(\tau)}{d \tau}=B_{2} z(\tau)+C_{2} u_{f}(\tau)
$$

Then the overall slow and fast subsystems of the normal SPF system are inferred as

$$
\begin{gathered}
\dot{x}_{s}(t)=\sum_{i=1}^{r} h_{i}\left\{A_{01} x_{s}(t)+B_{01} u_{s}(t)+C_{1} T_{r}\right\} \\
\frac{d z_{f}(\tau)}{d \tau}=\sum_{i=1}^{r} h_{i}\left\{B_{2} z(\tau)+C_{2} u_{f}(\tau)\right\}=B_{2} z(\tau)+C_{2} u_{f}(\tau)
\end{gathered}
$$

Remark 4: In order to simplify the model, we take the inputs, including $B_{01} u_{s}(t)$ and $C_{1} T_{r}$, of the (14a) as a generalized input $\bar{u}_{s}$, that is to say, $\bar{u}_{s}=B_{01} u_{s}(t)+C_{1} T_{r}$. The following system is then derived:

$$
\begin{aligned}
& \dot{x}_{s}(t)=\sum_{i=1}^{r} h_{i}\left\{A_{01} x_{s}(t)+\bar{u}_{s}(t)\right\} \\
& \frac{d z_{f}(\tau)}{d \tau}=B_{2} z(\tau)+C_{2} u_{f}(\tau)
\end{aligned}
$$

\section{Controller Design}

Now the wind turbine system is modeled as a singularly perturbed T-S fuzzy system. In fact, the model is composed of $r$ linearized subsystems. Based on the singularly perturbed T-S fuzzy model, the outputs could be predicted in time horizon $T$. In order to minimize the error $e(t)$ between the predicted outputs and the reference, a cost function of $e(t)$ is defined. Besides, variation of the input, $\dot{u}(t)$, will lead to overmuch transient load. Therefore, $\dot{u}(t)$ is also taken into the cost function. By minimization of the cost function, a sequence of future control inputs is derived. However, only the first input in the optimal sequence is sent into the plant, and the entire calculation is repeated at subsequent control intervals. The whole model predictive control strategy is presented in Fig. 1.

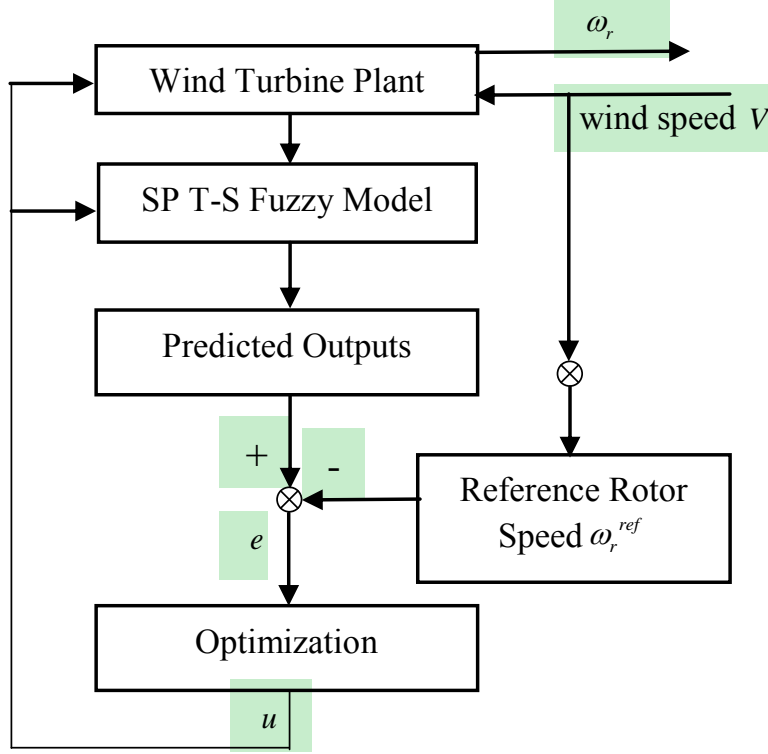

Fig. 1. SP T-S Fuzzy Model Predictive Control Structure.

Taking the slow subsystem for example, the model predictive control method ${ }^{[18]}$ is presented. The $j t h$ slow subsystem is given as follows:

$$
\begin{aligned}
& \dot{x}_{s}(t)=A_{s j} x_{s}+B_{s j} u_{s} \\
& y_{s}(t)=C_{s j} x_{s}
\end{aligned}
$$

with the cost function

$$
J_{s}=\int_{0}^{T}\left(X_{s}{ }^{\prime}\left(t_{i}+\tau \mid t_{i}\right) Q_{s} X_{s}\left(t_{i}+\tau \mid t_{i}\right)+\dot{u}_{s}{ }^{\prime}(\tau) R_{s} \dot{u}_{s}(\tau)\right) d \tau
$$

where $T$ is the prediction horizon, $X_{s}\left(t_{i}+\tau\right)$ is the state of augmented model as below:

$$
\begin{gathered}
X_{s}(t)=\left[\begin{array}{c}
\dot{x}_{s}(t) \\
y_{s}(t)-\omega_{r e f}(t)
\end{array}\right] \\
\dot{X}_{s}(t)=\left[\begin{array}{c}
\ddot{x}_{s}(t) \\
\dot{\dot{y}_{s}}(t)-\dot{\omega}_{r e f}(t)
\end{array}\right] \\
=\left[\begin{array}{ll}
A_{s j} & 0 \\
C_{s j} & 0
\end{array}\right]\left[\begin{array}{c}
\dot{x}_{s}(t) \\
y_{s}(t)-\omega_{r e f}(t)
\end{array}\right]+\left[\begin{array}{c}
B_{s j} \\
0
\end{array}\right] \dot{u_{s j}}(t)
\end{gathered}
$$

Remark 5: Since the wind speed is a continuous variable, it is reasonable to assume that $V(t)$ is derivable. Then, it is obvious to know that $\omega_{r e f}(t)=\frac{\lambda_{\text {opt }}}{R} V(t)$ is derivable, which means $\dot{\omega}_{\text {ref }}(t)$ exists.

The variation of the input, $\dot{u}_{j}(t)$, will lead to overmuch transient load and cost energy. As a consequence, instead of modeling the control signal, the continuous-time predictive control design will target the derivative of the control signal, $\dot{u}_{s j}(t)$, which will satisfy the property

$$
\int_{0}^{\infty} \dot{u}_{s j}(t) d t<\infty
$$

A set of Laguerre functions $L(\tau)$ is used as orthonormal basis functions ${ }^{[18]}$. According to [18], the derivative of the control signal can be described as:

$$
\dot{u}_{s j}(\tau) \approx \sum_{i=1}^{N} C_{i} l_{i}(\tau)=L^{\prime}(\tau) \varsigma
$$

where $\varsigma=\left[\begin{array}{llll}c_{1} & c_{2} & \cdots & c_{N}\end{array}\right]^{\prime}$ is the vector of coefficients as below:

$$
\begin{aligned}
& \varsigma=-\Omega^{-1} \Psi X_{s}\left(t_{i}\right) \\
& \Omega=\int_{0}^{T} \Phi(\tau) Q_{s} \Phi^{\prime}(\tau) d \tau+R_{L} \\
& \Psi=\int_{0}^{T} \Phi(\tau) Q_{s} e^{A_{s} \tau} d \tau \\
& \Phi^{\prime}(\tau)=\int_{0}^{\tau} e^{A_{s}(\tau-\gamma)}\left[B_{s 1} L_{1}{ }^{\prime}(\gamma) \quad \cdots \quad B_{s m} L_{m}{ }^{\prime}(\gamma)\right] d \gamma
\end{aligned}
$$


and the minimum of the cost function $J_{s \min }$ is:

$$
J_{s \min }=X_{s}^{\prime}\left(t_{i}\right)\left[\int_{0}^{T} e^{A_{s j}^{\prime} \tau} Q_{s} e^{A_{s} \tau} d \tau-\Psi^{\prime} \Omega^{-1} \Psi\right] X_{s}\left(t_{i}\right)
$$

So feedback gain matrix $K_{m s}$ is as below:

$$
K_{m s}=\left[\begin{array}{cccc}
L_{1}{ }^{\prime}(\tau) & 0 & 0 & 0 \\
0 & L_{2}{ }^{\prime}(\tau) & 0 & 0 \\
0 & 0 & \ddots & 0 \\
0 & 0 & 0 & L_{m}{ }^{\prime}(\tau)
\end{array}\right] \Omega^{-1} \Psi \triangleq\left[\begin{array}{ll}
K_{x s} & K_{y s}
\end{array}\right](27)
$$

Therefore, $u_{s j}(t)=-K_{m s} X_{s}(t)$, and $u_{s}(t)=\sum_{j=1}^{r} u_{s j}(t)$.

Similarly, we can get $u_{f}(t)$ using MPC for the fast subsystem. Then composite input $u(t)$ for the original system is obtained:

$$
u(t)=u_{s}(t)+u_{f}(t)
$$

\section{Example}

In this section, the parameters of the CART 3 located at the NREL are utilized to validate the control method proposed. The CART 3 parameters are summarized in Table 2.

The control objective is to optimize the wind power capture. Therefore, the tip speed ration should be fixed to its optimal value by maintaining the rotor speed $\omega_{r}$ at the optimal rotor speed $\omega_{r}^{\text {ref }}=\lambda_{\text {opt }} \frac{V(t)}{R}$.

Table 2. Wind Energy Conversion System Parameter

\begin{tabular}{|l|l|}
\hline Tip speed ratio $\lambda_{\text {opt }}$ & 5.8 \\
\hline Blade rotor $R$ & $21.65 \mathrm{~m}$ \\
\hline Air density $\rho$ & $0.98 \mathrm{Kg} \mathrm{m}^{-3}$ \\
\hline Gearbox ratio $i$ & 43.165 \\
\hline Wind rotor inertia $J_{r}$ & $3.88 \mathrm{Kg} \mathrm{m}^{2}$ \\
\hline Generator inertia $J_{g}$ & $0.22 \mathrm{Kg} \mathrm{m}^{2}$ \\
\hline Stator $d$ - axis inductance $L_{d}$ & $41.56 \mathrm{mH}$ \\
\hline Stator $q$-axis inductance $L_{q}$ & $41.56 \mathrm{mH}$ \\
\hline
\end{tabular}

\subsection{Constant Tracking Example}

The first example is to track a step signal as seen in Fig 2. The red solid line is the reference. The T-S MPC controlled results in blue are compared with the ones controlled by Nonlinear Feedback Linearization (NFL) method. Tracking errors are presented in Fig 3. It shows that rotor speed $\omega_{r}$ can reach the optimal value faster controlled by T-S MPC than that controlled by NFL controller.

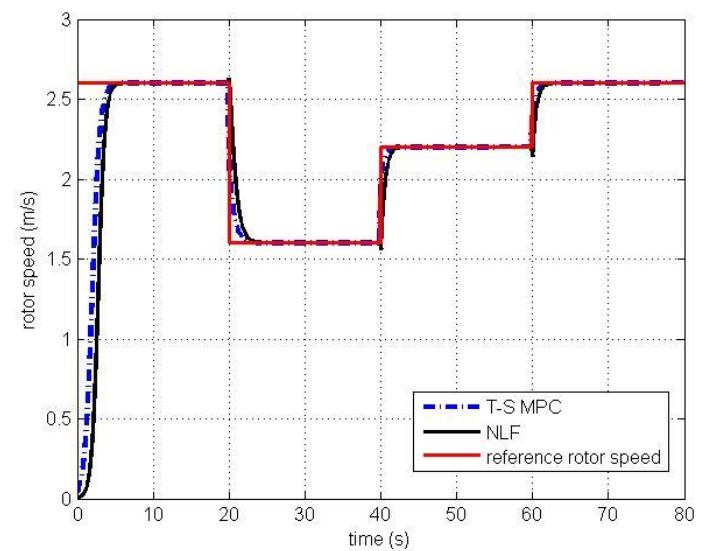

Fig 2: Constant Tracking

\subsection{Turbulence Tracking Example}

The T-S MPC controller is tested to track the wind turbulence. And the controlled results are shown in Fig 4. It can be seen that the rotor speed controlled by the T-S MPC can track the desired rotor speed much better than that of the NFL method.

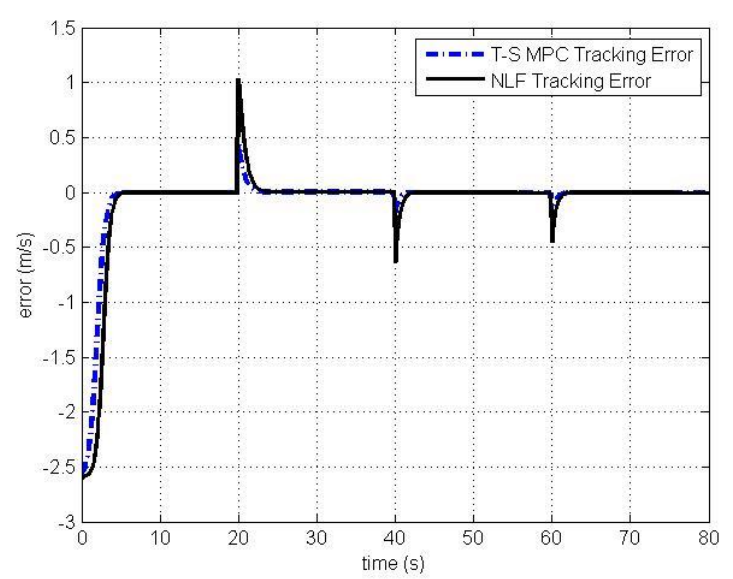

Fig 3: Constant Tracking Error

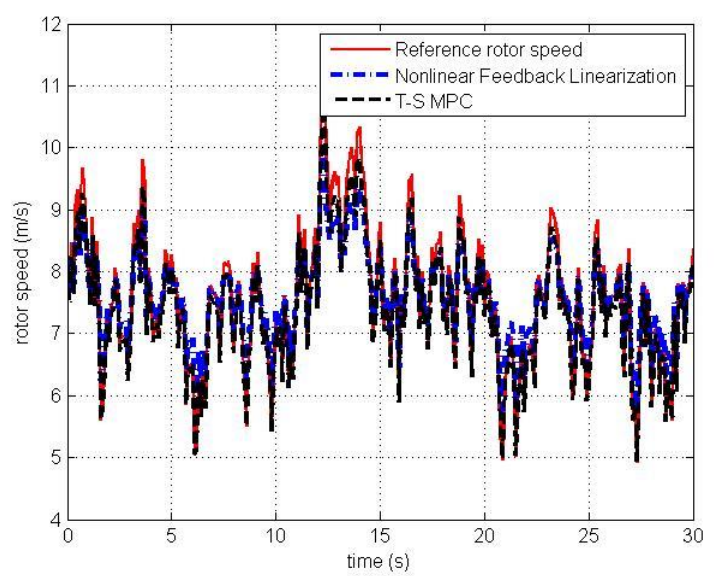

Fig 4: Wind Turbulence Tracking Results by T-S MPC and NFL 
The controllers performances are compared in Table 3 using the aerodynamic efficiency $\eta_{\text {aero }}$. It is defined as

$$
\eta_{\text {aero }}(\%)=\frac{\int_{t_{0}}^{t_{f}} P_{a} d t}{\int_{t_{0}}^{t_{f}} P_{a o p t} d t}
$$

where $P_{\text {aopt }}=\frac{1}{2} \rho \pi R^{2} C_{P_{\text {opt }}} v^{3}$ is the optimal aerodynamic power corresponding to the wind speed. Apparently, the method proposed in this paper improved the aerodynamic efficiency compared with the NFL method.

Table 3. Comparison of different controllers.

\begin{tabular}{|c|c|}
\hline & $\eta_{\text {aero }}(\%)$ \\
\hline NLF & 89.46 \\
\hline T-S MPC & 92.55 \\
\hline
\end{tabular}

\section{Conclusion}

In this paper, a singularly perturbed T-S model is proposed of wind energy conversion systems. The maximum point tracking problem is studied, and a T-S MPC method is developed. In the end, two numerical examples are carried out the compare the control strategy proposed with the nonlinear feedback linearization method. The results show that the T-S MPC brings better performance.

This work was jointly supported by the Natural Science Foundation of Jinling Institute of Technology (40620029), National Science Foundation of the Higher Education Institutions of Jiangsu Province (No.15KJB520010), Jiangsu Province's Natural Science Foundation (No.BK20171114), A joint prospective Industry-University-Research Collaboration project of Jiangsu Province (No.BY2016012-02), and Ministry of Education's Cooperative Education Project (No.201701056001, No.201602009006).

\section{References}

1. B. Boukhezzar, L. Lupu, H. Siguerdidjane, et al. Multivariable control strategy for variable speed, variable pitch wind turbines. Renewable Energy, 32(8):1273-1287 (2007).

2. A. Mechter, K. Kemih, M. Ghanes. Backstepping control of a wind turbine for low wind speeds. Nonlinear Dynamics, 84(4):2435-2445 (2016).

3. A. Bektache, B. Boukhezzar. Nonlinear predictive control of a DFIG-based wind turbine for power capture optimization. Int. J. Elec. Power, 101:92102 (2018).

4. S. M. R. Kazmi, H. Goto, H. J. Guo, et al. A Novel Algorithm for Fast and Efficient Speed-Sensorless
Maximum Power Point Tracking in Wind Energy Conversion Systems. IEEE T. Ind. Electron, 58(1):29-36 (2010).

5. D. C. Phan, S. Yamamoto. Rotor speed control of doubly fed induction generator wind turbines using adaptive maximum power point tracking. Eng., 111:377-388 (2016).

6. M. M. Hand, M. J. Balas. Non-Linear and Linear Model Based Controller Design for Variable-Speed Wind Turbines. (New York American Society of Mechanical Engineers, 1999)

7. X. Yao, C. Guo, Z. Xing, et al. Pitch regulated LQG controller design for variable speed wind turbine. International Conference on Mechatronics and Automation, 845-849 (2009).

8. X. Wang, S. Hou, S. Wang, et al. Multi-objective optimization torque control of wind turbine based on LQG optimal control. Chinese Control and Decision Conference, 405-408 (2013).

9. Y. M. Liu, E. Y. Yi, X. D. Wang, et al. Research on Capture the Maximum Wind Energy Base on Fuzzy PID Controller. App. Mech. \& Materials, 268270:1422-1425 (2013).

10. C. Xiao, L. Zhang, J. Yan. Fuzzy PID Controller for Wind Turbines. The Second International Conference on Intelligent Networks and Intelligent Systems.74-77 (2009).

11. K. Ro, H. H. Choi. Application of neural network controller for maximum power extraction of a gridconnected wind turbine system. Elec. Eng., 88(1):45-53 (2005).

12. T. Diveux, P. Sebastian, D. Bernard, et al. Horizontal axis wind turbine systems: optimization using genetic algorithms. Wind Energy, 4(4):151171 (2001).

13. B. Boukhezzar, H. Siguerdidjane. Nonlinear Control of a Variable-Speed Wind Turbine Using a Two-Mass Model. IEEE T. Energy Conver., 26(1):149-162 (2011).

14. X. Liu, X. Kong. Nonlinear Model Predictive Control for DFIG-Based Wind Power Generation. IEEE T. Auto. Sci. Eng., 11(4):1046-1055 (2014).

15. Y. Zhang, D. S. Naidu, C. X. Cai, and Y. Zou, Singular perturbation and Time Scales in Control Theories and Applications: An Overview 20022012, Inter. J. Infor. \& Sys. Sci., 9: 1-36 (2014).

16. D. S. Naidu, Singular Perturbation Methodology in Control Systems (Peter Peregrinus Ltd., 1988).

17. K. Xu. Singular Perturbation in the Control Systems (Science China Press, 1986).

18. L. Wang, Model Predictive Control System Design and Implementation Using MATLAB, (Springer, 2009). 